\title{
A acessibilidade no etourism: um estudo na ótica das pessoas portadoras de necessidades especiais
}

\author{
Cátia Filipa Macedo* Bruno Miguel Sousa** \\ Instituto Politécnico do Cávado e do Ave (Portugal)
}

\begin{abstract}
Resumo: As páginas web são, atualmente, o rosto de qualquer organização turística e representam a porta de entrada para novos consumidores. Por este motivo, verifica-se uma crescente preocupação em criar ferramentas digitais acessíveis que promovam a instituição, divulguem a sua oferta turística e fomentem o consumo. Por conseguinte, o uso das ferramentas digitais no turismo e, mais concretamente, no turismo direcionado para pessoas portadoras de necessidades especiais é de maior importância, no sentido de informar os potencias consumidores de um turismo verdadeiramente acessível a todos. O modo como as organizações utilizam as plataformas digitais, e mais especificamente os grupos hoteleiros/entidades de management, demonstram não cumprir as diretrizes de acessibilidade WCAG, havendo ainda um longo caminho a percorrer até ser possível afirmar um turismo de acesso universal. Para tal, deve haver um compromisso mais afincado das entidades reguladoras do setor. O presente estudo representa uma amostra importante na forma como são negligenciadas as questões ligadas à acessibilidade no eTourism, sendo uma área que carece de aprofundamento, pois a incorporação da acessibilidade na web, além de revelar um ganho social e inclusivo, impulsiona a atração de novos mercados e de projetos com maior qualidade. Os resultados obtidos parecem evidenciar que algumas organizações (no estudo consideradas) não cumprem os requisitos mínimos das diretrizes de acessibilidade WCAG, aspeto esse que aparentemente não constitui uma prioridade. As páginas web destas organizações expõem barreiras que impedem a inclusão digital.
\end{abstract}

Palavras-chave: Acessibilidade; eTourism; Marketing digital; Turismo para portadores de necessidades especiais; WCAG.

The accessibility in etourism: a study from the perpespective of people with specific needs

Abstract: Web pages are currently the new face of any Tourism organization. They represent the entrance door for new consumers. For this reason, there is a constant concern in creating accessible digital tools that could promote the institution, disclose its tourism offer and favor consumption. Thus, using digital tools in Tourism and, more concretely, in Tourism focused to people with special needs, is of great significance, in order to inform potential consumers of a tourism service accessible to all. The way the organizations make use of digital platforms, and more specifically hotels groups and management bodies, reveals that WCAG accessibility guiding lines are not followed, being still a long way to go before a universal accessible tourism can be effective. To do so, there might be a more rigorous compromise from the sector's regulatory authorities. The present study strongly suggests how are neglected the questions linked to accessibility in eTourism, this area deserving deeper insights. Indeed, incorporating accessibility in Web would stimulate the attraction of new markets and projects of higher quality, in addition to favoring a social and gathering gain. The results show that some organizations do not meet the minimum requirements of the accessibility guidelines, which is apparently not a priority. The web pages of these organizations expose barriers that prevent digital inclusion.

Keywords: Accessibility; eTourism; Digital marketing; Tourism to people with special needs; WCAG.

\footnotetext{
* Instituto Politécnico do Cávado e do Ave (Portugal); E-mail: cfilipa_7@hotmail.com

* Instituto Politécnico do Cávado e do Ave (Portugal); E-mail: bsousa@ipca.pt
} 


\section{Introdução}

O turismo é, atualmente, o setor da sociedade que apresenta maior crescimento em todo o mundo. Associada à deslocação de pessoas por razões de ócio, trabalho ou saúde, a atividade turística apresenta-se envolvida numa complexa malha de relações, obrigando a constantes mudanças no modus operandus das organizações. As sociedades desenvolvidas, impulsionadas pela mais recente tecnologia, além de promover a movimentação de pessoas e a procura de produtos/serviços turísticos, potencia novos desafios e maiores exigências (Schlüter \& Var, 1988; Yuksel, Yuksel \& Bilim, 2010; Altinay, Saner, Bahçelerli \& Altinay, 2016).

Neste sentido, a gradual transformação dos meios tecnológicos abriu novas perspetivas e diferentes formas de comunicar e de apresentar o produto/serviço turístico. Estes mecanismos permitiram quebrar a estrutura da cadeia turística, ao possibilitarem encurtar a distância entre o promotor e o consumidor do produto/serviço turístico. Nota-se, cada vez mais, que é o próprio consumidor que se informa e se organiza, fazendo as suas reservas e aquisições de produtos diretamente através do canal web. Assim, as ferramentas digitais permitem ter um conhecimento mais rápido, alargado e abrangente das ofertas turísticas nas diferentes partes do globo (Buhalis, Darcy \& Ambrose, 2012).

O presente estudo pretende, assim, averiguar o uso das ferramentas digitais no setor do turismo, centrando o estudo na ótica de pessoas portadoras de necessidades especiais. A criação de destinos turísticos acessíveis passa por um acesso à informação universal. Com as significativas alterações aos meios de comunicação e de informação, as empresas devem, ainda, ajustar-se na conceção das ferramentas digitais, de forma a conseguir incluir todas as pessoas no seu uso, eliminando as barreias existentes à comunicação e interação entre empresa e consumidor. A literatura disponível abre caminho a que novos estudos sejam desenvolvidos no sentido de se verificar se os websites (não) apresentam conteúdos acessíveis a todos os utilizadores.

\section{Revisão de literatura}

\subsection{O marketing digital no turismo - etourism}

Os novos tempos trouxeram maior desenvolvimento tecnológico e formas inovadoras para o marketeer. Segundo Chaffey, Ellis-Chadwick, Mayer e Johnston (2006), com a era digital, a internet e outros meios de comunicação digital alancaram novos termos de marketing como, por exemplo, "Internet Marketing", "E-marketing", "Digital Marketing", "E-business" e "E-commerce". Atualmente, as tecnologias já não são novas, mas têm confluído e registado um impacto significativo na sociedade e, substancialmente, nas práticas do marketing. Assim, denota-se que a era digital alancou novos consumidores, mais informados e conectados, que impulsiona, dia após dia, a criatividade dos marketeers.

O rápido e complexo desenvolvimento tecnológico tem revolucionado a indústria do turismo e permite ao eTourism o processamento e a comunicação de dados eficazes, que reúne esforços para combater as suas implicações baseadas em aspetos como a interoperabilidade, design e análise e modelagem dos sites (Buhalis, Leung \& Law, 2011). Ao longo dos últimos 6 anos, os estudos desenvolvidos sobre a adoção do marketing digital na hotelaria vieram impulsionar o estudo do comportamento dos consumidores na indústria do turismo. Refira-se alguns estudos de: Shuai e Wu (2011), detetaram que os hotéis em Taiwan usavam os sites, essencialmente, para fornecer informações e transações, em vez de interagir com os clientes, demonstrando uma presença simples na internet e um desaproveitamento da interatividade e funcionalidade do site; El-Gohary (2012), descobriu que a adoção do marketing digital nos hotéis do Egipto está relacionada com fatores internos e externos (tais como: formação dos colaboradores, recursos disponíveis, cultura organizacional, financiamentos, facilidade do uso, compatibilidade, pressões competitivas, influências políticas, tendências do mercado e orientação cultural); Escobar-Rodríguez e Carvajal-Trujillo (2013), verificaram que os hotéis espanhóis começaram a implementar ferramentas relacionais orientadas para o cliente, e que até então os sites eram meramente informativos; Li, Wang e Yu (2015), analisaram as atividades de marketing dos hotéis dos Estados Unidos da América (EUA) e notaram que os seus sites apenas informavam e não interagiam com os consumidores, necessitando de construir relacionamentos e interatividade com estes; e Stephen (2016), agregou pesquisas de diversos autores e identifica cinco temas relevantes para a pesquisa do comportamento do consumidor no marketing digital (os quais: cultura digital do consumidor, publicidade, impactos de ambientes digitais, dispositivos móveis e boca-a-boca [WOM] online). 
Seguindo a perspetiva de Buhalis e Law (2008), o eTourism centraliza-se nas tecnologias focadas no consumidor e que apoiam as empresas a interagir com eles de forma dinâmica, reinventando produtos/ serviços turísticos de forma proativa e reativa, fundamentais para a competitividade no mercado.

\subsection{O turismo para portadores de necessidades especiais}

Já há algum tempo que o marketing de nichos ou target marketing ou marketing focalizado ou marketing concentrado ou micromarketing é uma estratégia que utiliza a diferenciação, o posicionamento, a rentabilidade, o foco/a especialização, o relacionamento de longo prazo e a reputação como elementos fundamentais para o sucesso das empresas em mudança (Dalgic \& Leeuw, 1994; Phillips \& Peterson, 2001). Atendendo às necessidades e preferências dos turistas, que começaram a desvalorizar essas "máquinas de viagens" e a procurar experiências individualizadas, impulsionando serviços personalizados, o turismo de nicho oferece níveis crescentes de experiências (Robinson \& Novelli, 2005), podendo mesmo criar laços e distinções sociais, associados aos prazeres emocionais e desejos (Giddens, 1990; Beck, 1991). Os padrões de consumo turístico originaram um mercado mais segmentado, especializado e sofisticado que envolveram o desenvolvimento de nichos para responder às necessidades dos consumidores (Robinson \& Novelli, 2005). Porém, nem sempre as expectativas dos turistas são superadas, geralmente para as pessoas com necessidades especiais cuja má aplicação dos padrões de acessibilidade limitam a sua participação na atividade turística.

Muitas vezes, o termo acessibilidade confunde-se com a dificuldade sentida em meios físicos, associando-o a pessoas com deficiências ou incapacidades. Contudo, a acessibilidade incorpora o meio edificado, os transportes e as tecnologias da informação e da comunicação, tornando-se indispensável para que todos os cidadãos possam exercer dos seus direitos de cidadania e da participação na vida social, cultural, económica e política. Darcy (2001, p. 74) reforça esta ideia dizendo que "o acesso não é apenas sobre edifícios; um ambiente verdadeiramente acessível é aquele em que uma pessoa com deficiência pode expressar livremente a sua independência e aquele em que um impedimento à integração é removido". São várias as denominações utilizadas por autores como Alles (2009), Darcy, Carmeron e Pegg (2011), Hoyo e Valiente (2010), Gouveia, Mendes e Simões (2011) e Montes e Aragall (2009) - "Turismo Acessível" e "Turismo Social", dando origem ao "Turismo Inclusivo" e, por conseguinte, ao denominado "Turismo para Todos". Neste sentido, e tratando-se de um tipo de turismo destinado a um público-alvo muito específico, que procura atender às necessidades especiais de pessoas portadoras de alguma deficiência, incapacidade ou limitação, entende-se ser possível enquadrar o conceito de "Turismo para Portadores de Necessidades Especiais".

A divulgação das condições de acessibilidade na oferta turística incentiva, potencialmente, a procura dos destinos. $\mathrm{O}$ facto de um destino procurar soluções de modo a satisfazer as necessidades e preferências de um turista, independentemente da natureza e grau de incapacidade de que é portador, fomenta um espírito de responsabilidade social e corporativo, contribuindo para um destino diferenciado e competitivo e, ao mesmo tempo, sustentável. A conviç̧ão de que apenas as pessoas portadoras de deficiência beneficiam da acessibilidade está profundamente errada. Até porque, são vários os tipos de deficiências, incapacidades e limitações que carecem de condições acessíveis para o consumo turístico. Por exemplo, os acompanhantes (familiares ou amigos) que escolhem um destino e programam as férias tendem a considerar os níveis de acessibilidade e barreiras que possam existir durante a sua estada. Também, as crianças, para quem a acessibilidade significa segurança e autonomia, e os familiares que as acompanham, que precisam de condições especiais para determinadas tarefas (amamentação, muda de fraldas, hora da sesta, entre outras). E ainda, os idosos, que são um grupo diversificado e com maior incidência de deficiências e incapacidades, nomeadamente ao nível motor, visual, auditivo e mental, e com limitações na saúde derivadas de problemas cardíacos ou respiratórios, diabetes, obesidade, alergias.

Face ao exposto, pode concluir-se que a acessibilidade incute um envolvimento proativo e direto com o cliente, conseguido após um conhecimento do seu perfil e das suas características. Em Portugal, o Inquérito Nacional às Incapacidades, Deficiências e Desvantagens (INR, 1996), publicado pelo Secretariado Nacional para a Reabilitação e Integração das Pessoas com Deficiência (SNRIPD), agora Instituto Nacional para a Reabilitação, I.P. (INR), ditava um valor global de $9,16 \%$ da população com incapacidades, deficiências e desvantagens - que representava quase um milhão de pessoas -, em categorias como: ver; ouvir; falar; outras incapacidades de comunicação; cuidado pessoal; locomoção; tarefas diárias; incapacidades face a situações (que decorrem da dependência e da resistência física, por exemplo ventiladores respiratórios); e ainda ao nível do comportamento (educação, segurança pessoal, relacionamento familiar e atividade profissional). 
Contudo, os Censos de 2001 não monopolizavam o ponto de vista das incapacidades, mas das deficiências (visual, motora, auditiva, mental, paralisia cerebral ou outras), ficando por rastrear muitas outras causas e, assim, não se avaliou com rigor a funcionalidade dos cidadãos. Ainda assim, os dados revelaram que 636059 pessoas tinham algum tipo de deficiência, ou seja, 6,1\% da população (Gonçalves, 2003). Um estudo intitulado "Saúde e Incapacidades em Portugal 2011" apurou que cerca de 18\% das pessoas tinham, simultaneamente, problemas de saúde prolongados e dificuldades na realização de pelo menos uma das seis atividades básicas do dia-a-dia mencionadas nos censos - ver, ouvir, andar ou subir degraus, memória/concentração, tomar banho ou vestir-se sozinho, compreender os outros ou fazer-se compreender (INE, 2012). No entanto, é bem visível a falta de informação sobre a realidade de vida das pessoas com deficiências e/ou incapacidades e a forma como os dados estatísticos se foram dissipando.

A nível europeu, o projeto OSSATE estimou que 127,5 milhões de pessoas (entre sete segmentos de pessoas com deficiências e população idosa) carecem da acessibilidade, representando $27 \%$ da população europeia (Buhalis, Eichhorn, Michopoulou \& Miller, 2005). Mais tarde, surge a "Estratégia Europeia para a Deficiência 2010-2020: Compromisso renovado a favor de uma Europa sem barreiras" referindo que um em cada seis cidadãos (cerca de 16,7\% da população) - ou seja, cerca de 80 milhões de pessoas - tem algum tipo de incapacidade (Comissão Europeia, 2010). Em 2012, a "Pesquisa Europeia de Integração Social e Saúde (EHSIS)" apontou para a existência de 70 milhões de pessoas com incapacidades de idade superior a 15 anos, igual a $17,6 \%$ da população.

Já a nível mundial, o relatório "World report on disability", publicado pela Organização Mundial da Saúde com a parceria do Banco Mundial (WHO, 2011, p. 44), indica que "cerca de 785 (15,6\% de acordo com a Pesquisa Mundial de Saúde) a 975 (19,4\% de acordo com a Carga Global de Doenças) milhões de pessoas com 15 anos ou mais vivem com alguma incapacidade, com base nas estimativas populacionais de 2010 (6,9 bilhões de habitantes, com 1,86 bilhões de pessoas menos de 15 anos). Entre elas, a Pesquisa Mundial de Saúde estima que 110 milhões de pessoas (2,2\%) enfrentam dificuldades funcionais bastante significativas, enquanto que a Carga Global de Doenças estima que 190 milhões de pessoas $(3,8 \%)$ possuem "incapacidades graves" - o equivalente às incapacidades inferidas para condições tais como quadriplegia, depressão grave, ou cegueira". O mesmo estudo revela que, ao incluir as pessoas com idade inferior a 15 anos, os números ultrapassam os mil milhões de pessoas que têm algum tipo de incapacidade - i.e., cerca de $15 \%$ da população mundial. Mas estes valores merecem uma reflexão sobre as potencialidades do mercado interno. E, também, porque o envelhecimento da população tem sido cada vez mais acentuado.

Neste sentido, impõe-se a abordagem específica da acessibilidade no turismo, e o correspondente derrube de barreiras físicas, sensoriais, informativas e comunicativas, que tem por base os princípios e fundamentos do design universal, até porque a normalização da incapacidade, do envelhecimento e do acesso potenciaria a criação de ambientes turísticos capacitadores (Darcy, Ambrose, Schweinsberg \& Buhalis, 2011; Darcy \& Dickson, 2009). A acessibilidade no sistema do turismo interage com todos os componentes da cadeia de necessidade de informação dos clientes, bem como o acesso físico (transportes e destino) e o acesso à informação. Apesar disso, as barreiras ao acesso são complexas e não podem ser reduzidas, apenas, pelas construções ambientais e informação física (Buhalis et al., 2005). Assim, a informação disponibilizada deve ser de alta qualidade, precisa, verdadeira e detalhada, e deverá ser acedida em diferentes formatos e suportes, nomeadamente via internet, mensagens escritas, brochuras, catálogos, braille, entre outros (Vos \& Michopoulou, 2006). Também, a informação sobre a acessibilidade deve seguir parâmetros e padrões standardizados, pois a par da normalização universal, cada região ou país tem as suas leis, regulamentos e orientações internas, as quais revelam grandes disparidades.

\section{Figura 1: Símbolo de Acessibilidade na Web}

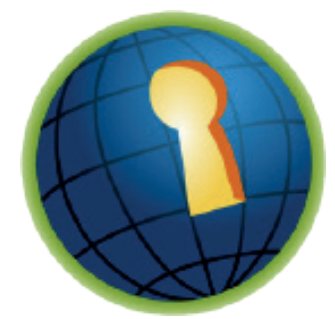

Fonte: Unidade Acesso (2017) 
Ao complementarem o projeto One Stop Shop for Accessible Tourism in Europe (OSSATE), no relatório sobre o "Inventário de esquemas de acessibilidade e conjuntos de dados no setor de turismo da UE", Vos e Michopoulou (2006) referem que não existe o esquema de informação acessível, mas antes diversos esquemas que variam consoante as infraestruturas aplicadas, os métodos e critérios utilizados e a forma de apresentação das informações. Há uma tendência generalizada em considerar que apenas as pessoas com mobilidade reduzida necessitam de informação acessível, demonstrando que, apesar de se utilizar distintas formas de apresentação, nem toda a informação é passível de acessibilidade. Observaram, ainda, que grande parte da informação sobre a acessibilidade é disponibilizada através dos websites, carecendo uma percentagem substancial do selo de acessibilidade eletrónica (figura 1), o denominado "Símbolo de Acessibilidade na Web" (Unidade Acesso, 2017).

\subsection{A acessibilidade no etourism}

Recorde-se de Stephen W. Hawking, físico e cosmólogo conceituado, portador de esclerose lateral amiotrófica que, através de um pequeno computador adaptado à sua cadeira de rodas elétrica, com sintetizador de voz, conseguiu ganhar autonomia e independência. As TIC, quando acessíveis, oferecem formas de interação com o conteúdo digitalizado e facilitam a comunicação entre as pessoas portadoras ou não de necessidades especiais, permitindo melhorias na qualidade de vida ao ampliar as suas independências e habilidades. No entanto, caso as TIC não sejam acessíveis, podem espelhar a autoestima dessas pessoas, pelo isolamento e discriminação das mesmas.

O acesso à informação (abordado no projeto OSSATE) sofreu significativas alterações com a criação da internet, pois a web elimina barreiras à comunicação e à interação entre as pessoas. Todavia, os sites, os aplicativos, as tecnologias ou as ferramentas quando mal concebidos podem potenciar a criação de barreiras e contribuir para a exclusão de pessoas no uso da web. (W3C, 2017). De acordo com o artigo $9^{\circ}$, alínea g) da Convenção sobre os Direitos das Pessoas com Deficiência, o acesso às TIC, incluindo a web, é reconhecido como um direito humano básico e a sua acessibilidade proporciona um acesso universal e a igualdade de oportunidades a pessoas com necessidades especiais (Assembleia da República, 2009, p. 4910; W3C, 2017). O termo "Acessibilidade na Web" é utilizado quando os recursos disponíveis na web são acedidos e manuseados por qualquer pessoa, portadora ou não de necessidades especiais, permitindo que todos tenham acesso igual a informações e funcionalidades na web (EU, 2014; WHO, 2017). Considerando a importância da acessibilidade na web, torna-se fundamental a harmonização de modelos e de regras da acessibilidade no desenvolvimento de sites, aplicativos, tecnologias ou ferramentas. O World Wide Web Consortium, W3C - consórcio internacional composto por pessoas da indústria, organizações de deficiência, governo e laboratórios de pesquisa de todo o mundo (W3C, 2017) -, em abril de 1997, lançou a iniciativa Web Accessibility Initiative, WAI, com o objetivo de orientar a acessibilidade na $w e b$, com o desenvolvimento de estratégias, diretrizes e recursos.

Com a iniciativa WAI, verifica-se que a interdependência de vários componentes permite melhorar consideravelmente a acessibilidade na web (Figura 2). Esses componentes incluem: o conteúdo; os usuários que usam browsers, media players e tecnologias assistidas (leitores de tela, teclados alternativos, software interativo, etc.); e os developers que usam ferramentas de criação e ferramentas de avaliação. Segundo a WAI, a acessibilidade na web incluiu quatro guias de orientação (W3C, 2017): WCAG (Web Content Accessibility Guidelines); ATAG (Authoring Tool Accessibility Guidelines; UAAG (User Agent Accessibility Guidelines); e WAI-ARIA (Accessible Rich Internet Applications Suite).

Além da importância destes guias de orientação para a acessibilidade na web, é fundamental a avaliação e a validação da acessibilidade através de ferramentas automáticas, geralmente mais rápidas mas incapazes de identificar todos os aspetos da acessibilidade, ou de ferramentas manuais, que ajudam a garantir a clareza da linguagem e a facilidade de navegação (Conforto \& Santarosa, 2002). Alguns exemplos de ferramentas de avaliação automática para os princípios e as diretrizes WCAG são os validadores: AccessMonitor; Wave - Web Accessibility Tool; Total Validator; e FAE - Functional Accessibility Evaluator (comAcesso, 2017; W3C, 2017). Apoiando o contexto atual mencionado pela Comissão Europeia - ditando que o número de pessoas com necessidades especiais tende a aumentar para 120 milhões até 2020 - é aprovada, a 14 de setembro de 2017, a "Lei Europeia da Acessibilidade" a qual criará requisitos comuns de acessibilidade a aplicar em determinados produtos e serviços (entre eles, serviços de turismo e websites) às pessoas com necessidades especiais (EU, 2017a; DN, 2017). Ainda, durante este período de dois anos, assistiu-se a um acordo tripartido (entre negociadores do Parlamento Europeu, do Conselho e da Comissão) onde foram redigidas as primeiras regras para "tornar os sítios web e as aplicações móveis dos organismos do setor público mais acessíveis, em especial para as pessoas 
Figura 2: Os componentes essenciais da acessibilidade da web e a sua interdependência

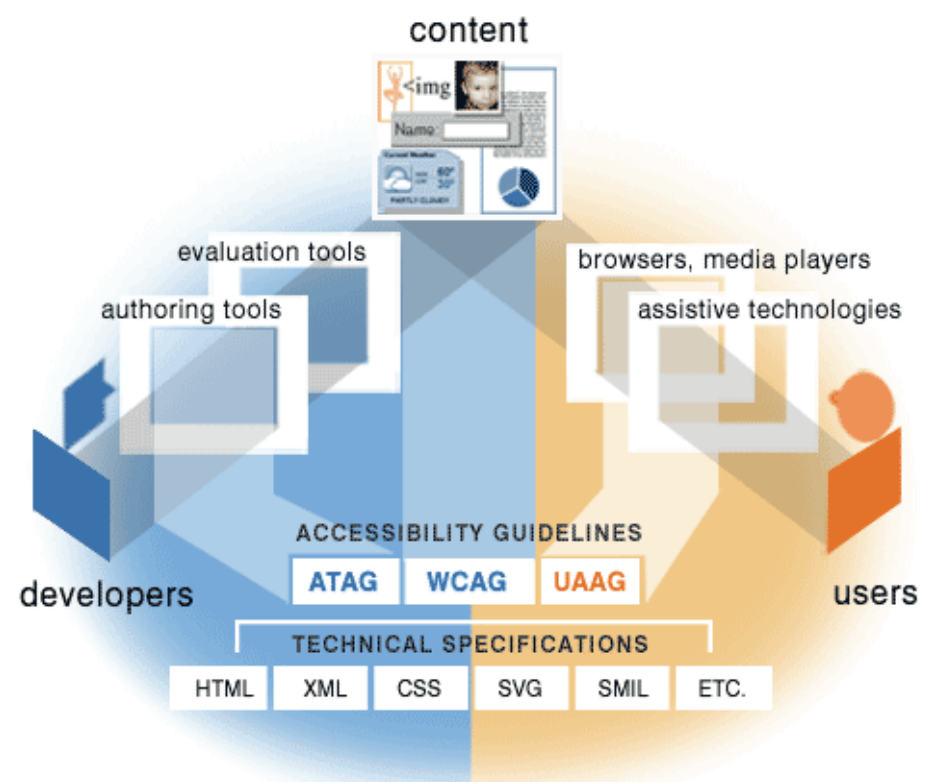

Fonte: W3C (2017).

cegas, surdas ou com deficiência auditiva" (EU, 2017b) - decretada pela Diretiva (UE) 2016/2102 do Parlamento Europeu e do Conselho, de 26 de outubro de 2016, relativa à acessibilidade dos sítios web e das aplicações móveis de organismos do setor público (EUR-LEX Europe, 2016). Tem-se verificado que o uso das plataformas digitais é transversal a todos os cidadãos e em todos os setores de atividade.

De acordo com Buhalis e Law (2008), a indústria do turismo deve estar consciente de que as pessoas com necessidades especiais representam um nicho de mercado em constante crescimento e que, para expandir o negócio e melhorar o seu serviço, a sua presença na web deve responder às necessidades desse nicho e projetar sites que abordam a inclusão. A web alterou toda a indústria do turismo e as suas disparidades demonstram ter um impacto significante na produção e usufruto dos consumidores, denotando que a inacessibilidade na web incapacita os consumidores de usufruírem de um serviço, de obterem informações ou de reservarem uma viagem (Minghetti \& Buhalis, 2009; Puhretmair, \& Nussbaum, 2011; Michopoulou \& Buhalis, 2014). O desenvolvimento das TIC oferece novas e impressionantes vantagens para as pessoas com necessidades especiais e a indústria do turismo, para atrair este nicho de mercado, necessita que as suas informações, publicadas em páginas $w e b$, sejam acessíveis a eles e que sejam confiáveis, precisas e atualizadas (Buhalis \& Michopoulou, 2011; Puhretmair, \& Nussbaum, 2011; Altinay, Saner, Bahçelerli \& Altinay, 2016).

Michopoulou e Buhalis (2011, p. 291; 2013, p. 231), nos seus estudos, mencionam quatro desafios "aprimorados", "intensificados" e "genéricos no setor do turismo" aquando a criação de websites acessíveis, a citar: interoperabilidade, ou seja a capacidade de um sistema para interagir e comunicar com outro (comunicação em rede); integração de conteúdo, um fator determinante na decisão de compra dos consumidores, através do detalhe, profundidade e tipo de informação disponível; personalização, i.e., gerar conteúdos individualizados, permitindo que cada utilizador especifique os seus requisitos e interesses de cada um no acesso às informações necessárias e no formato desejado; e design acessível, considerar o layout dos websites e eliminar elementos como o baixo contraste de cores, os gráficos e as animações.

Os resultados da usabilidade de Michopoulou e Buhalis (2014) revelaram 20 requisitos essenciais para a acessibilidade no eTourism, baseados em opções de impressão, flexibilidade de interface e apresentação de conteúdo/informação. No seu estudo estes autores defendem a alteração do tamanho da fonte e ícones nas páginas da web; a automatização constante da entrada de dados; a adição de pequenas notas explicativas; a simplificação dos mecanismos de seleção e anexação de mapas e de imagens; a integração de filtros de acessibilidade (classificados alfabeticamente) com outros filtros turísticos em todo o processo 
de busca; o desenvolvimento de versões multilingues para um website; a inclusão de uma secção de fórum; e a versatilidade das páginas que devem estar disponíveis em formatos acessíveis e imprimíveis.

No entanto, ao disseminar a informação sobre os produtos e serviços turísticos acessíveis, a acessibilidade no eTourism poderá beneficiar todas as pessoas, com ou sem necessidades especiais, isto porque o diálogo inter-relacional entre as empresas e os indivíduos permitirá o aumento do poder do turismo, reduzindo a exclusão social (Eichhorn, Miller, Michopoulou \& Buhalis, 2008; Buhalis \& Michopoulou, 2011).

A adoção dos princípios do design universal permitirá aos utilizadores o livre acesso, sem a necessidade de adaptação ou de design especializado. Do mesmo modo, aos destinos turísticos potenciará um aumento significativo do mercado, atenuando a sazonalidade e apoiando a competitividade dos diferentes destinos (Buhalis, 2000; Vila, Darcy \& González, 2015; Michopoulou, Darcy, Ambrose \& Buhalis, 2015). Buhalis, Darcy e Ambrose (2012), no livro publicado sobre boas práticas do turismo acessível, apoiam a execução de um processo estrategicamente planeado, por um conjunto de fatores interligados (políticas e estratégias, redes e parcerias, cadeia de valor do turismo acessível e desenvolvimento de produtos e destinos), de forma a que seja possível fornecer experiências de turismo acessíveis.

No entanto, e apesar dos estudos realizados sobre a utilização dos consumidores na web (Grupo Marktest, 2017 e 2018; travelBI, 2018), a informação digital nem sempre está acessível a todos os utilizadores e a sociedade futura deverá procurar ser mais inclusiva, permitindo o acesso a todos os cidadãos, independentemente das suas diferenças. O setor do turismo deve melhorar o planeamento e projetar, operar e fornecer experiências acessíveis a todos os cidadãos, enfatizando as necessidades destes, conseguidas pela aplicação dos princípios do design universal e dos valores de independência, equidade e dignidade (Darcy \& Dickson, 2008). Além disso, a informação através dos canais web devem ser disponibilizados de forma detalhada, fiável e de fácil manejo. A promoção da informação turística acessível do destino Portugal é disponibilizada pelas entidades "Portugal Acessível by Associação Salvador ", "Visit Portugal" e "Accessible Portugal". Esta última está prestes a lançar a "TUR4all Portugal", uma plataforma e aplicação móvel de informação e divulgação da oferta turística acessível, em Portugal, de forma a captar um novo mercado e a melhorar as experiências dos turistas com necessidades especiais. Esta aplicação, já em vigor no país vizinho, conta com o apoio da Fundação Vodafone Portugal, do Turismo de Portugal e da European Network for Accessible Tourism (ENAT).

Apesar de todo o esforço realizado por entidades públicas e privadas, que procuram promover e desenvolver guias de acessibilidade (como por exemplo, "Guia de Boas Práticas de Acessibilidade na Hotelaria", "Guia de Boas Práticas de Acessibilidade -Turismo Ativo" e "Guia de Boas Práticas de Acessibilidade - Comunicação inclusiva em Monumentos, Palácios e Museus"), livros de apoio ao desenvolvimento de destinos turísticos ("Manual de Gestão de Destinos Turísticos Acessíveis"), programas estratégicos ("All for All-portuguese tourism") e projetos de sensibilização e com planos de ação ("Praia Acessível, Praia para Todos" e "Concelho Mais Acessível") - Turismo de Portugal (2018) e INR (2018) -, é insuficiente uma ação isolada das entidades e é imprescindível a cooperação entre todas para alcançarem o sucesso da acessibilidade.

Em termos internacionais, são várias as entidades que procuram implementar boas práticas de acessibilidade. A ENAT, direcionada para organizações que pretendem criar e desenvolver práticas para o turismo acessível, disponibiliza informação útil para melhorar a acessibilidade de informações turísticas, transportes, infraestruturas, design e serviços (ENAT, 2017). Na página oficial da ENAT ${ }^{4}$ é possível aceder às cidades europeias acessíveis que cumprem os requisitos do prémio "European Award for Accessible Cities", premiadas desde 2010, que inclui o melhoramento da acessibilidade de informação e de comunicação, incluindo as TIC.

No mesmo sentido, o "Europe for All", defendido pelo projeto europeu OSSATE, mencionado anteriormente, procura ajudar os consumidores a encontrar informação sobre a acessibilidade, de forma a planearem com facilidade e confiança as suas viagens, e melhora as comunicações entre os operadores turísticos e os clientes, fornecendo informações rigorosas e fiáveis sobre a acessibilidade de locais e serviços. Buhalis, Darcy e Ambrose (2012) e Gouveia et al. (2011) mencionam diversos projetos que retratam as boas práticas para a acessibilidade. No caso concreto da acessibilidade online, vários estudos têm sido desenvolvidos de forma a apoiar o conteúdo turístico acessível, a saber: Xiong, Cobanoglu, Cummings e DeMicco (2009), descobriram uma baixa acessibilidade nos websites de hotelaria nos EUA e a falha no fornecimento de textos alternativos em conteúdo não textual; Mills, Han e Clay (2008), detetaram as barreiras para utilizadores com incapacidades visuais, com falha no contraste de informação e na ausência de textos alternativos; Han e Mills (2006), ao questionarem os utilizadores, encontraram problemas como o excesso de pop-ups, a não leitura de tecnologias assistidas e as sucessivas reconstruções dos websites; Alexandre (2012), descobriu uma baixa acessibilidades nos websites de hotelaria em Lisboa e 
analisou a perspetiva dos responsáveis para a inclusão de turistas com necessidades especiais; e Castro (2017), analisou a acessibilidade física e online das atrações turísticas do Porto.

Do exposto, pode-se concluir que a acessibilidade no eTourism tem uma importância crucial na promoção e divulgação turística, no que respeita aos direitos universais dos cidadãos. Os estudos supracitados demonstram que esta temática assumiu já uma posição central em muitos países desenvolvidos que, na procura de soluções para a inclusão, produzem legislação nesse sentido. Assim, pode-se afirmar que um país acessível a todos é um país moderno, atrativo, inclusivo e socialmente responsável.

\section{Metodologia}

Considerando a crescente importância das plataformas digitais na promoção e divulgação de produtos/serviços turísticos, pretende-se com este estudo perceber de que forma as organizações abordam o marketing digital, mais especificamente, no que respeita ao marketing utilizado no turismo para portadores de necessidades especiais. Procura-se adquirir uma visão abrangente dos websites das cinco maiores cadeias hoteleiras, em Portugal, quanto à utilização dos padrões de acessibilidade e, assim, encontrar respostas relevantes e precisas quanto à questão primordial desta investigação:

De que modo as organizações utilizam as ferramentas digitais para pessoas portadoras de necessidades especiais?

A partir do objetivo geral da investigação foram definidos vários objetivos específicos que serviram de linhas orientadoras para o desenvolvimento do presente estudo, a saber: conhecer e discutir os conceitos de marketing digital e turismo de nichos; compreender o nicho de mercado - turismo para portadores de necessidades especiais; explorar os conceitos de acessibilidade e de inclusão digital (conhecer e compreender o atual panorama dos processos de acesso às plataformas digitais por parte dos portadores de necessidades especiais); conhecer estratégias, recursos e materiais que facilitem a construção de soluções multimédia acessíveis; analisar cinco grupos hoteleiros/entidades de management do ranking nacional e detetar quais possuem website com o respetivo símbolo de acessibilidade, bem como as boas práticas e as falhas da sua utilização; analisar os websites e avaliar os requisitos de acessibilidade, utilizando a ferramenta de análise automática e de uma análise manual; e desenvolver recomendações essenciais para a aplicação de requisitos mínimos de acessibilidade na web.

Uma vez definido o tema em estudo e delineados os principais objetivos, passou-se à recolha de dados. Numa primeira fase realizou-se uma pesquisa para identificar as cinco maiores cadeias hoteleiras em Portugal, de acordo com a $12^{\text {a }}$ edição do Atlas da Hotelaria 2017 (Deloite, 2017). Assim, serão analisados os seguintes grupos hoteleiros/entidades de management, de acordo com a sua posição no ranking: $1^{\circ}$ Pestana Hotels \& Resorts/Pousadas de Portugal; $2^{\circ}$ Vila Galé Hotéis; $3^{\circ}$ Accor Hotels; $4^{\circ}$ Tivoli Hotels \& Resorts; $5^{\circ}$ Marriott Hotels \& Resorts. Da análise detalhada da informação recolhida de cada organização, foi conferido se possui website com o respetivo símbolo de acessibilidade. Ressalva-se que esta análise foi efetuada no início de janeiro de 2018, podendo eventualmente ter ocorrido algumas alterações.

Inicialmente, foi necessário compilar os links de cada página principal dos cinco websites, pelo facto de se considerar que a página principal do website é o primeiro contacto com o utilizador e determina o acesso de utilizadores com necessidades especiais às restantes páginas do website. Na observação dos grupos hoteleiros/entidades de management, deixa-se uma nota para as Pousadas de Portugal que apenas são consideradas as que se encontram sob gestão do grupo Pestana Hotels \& Resorts, por isso, nesta investigação, será analisado apenas o link https://www.pestana.com/pt. À posteriori, utilizou-se uma ferramenta de análise automática e fez-se uma análise manual. No presente estudo, optou-se pelo validador automatizado AccessMonitor que monitoriza a aplicação das diretrizes de acessibilidade nos conteúdos HTML de um website, usando como referência a versão 2.0 das diretrizes de acessibilidade para o conteúdo web, WCAG (Web Content Accessibility Guidelines) do W3C - WCAG 2.0.

\section{Resultados}

Numa primeira análise, os resultados obtidos através da avaliação automática realizada aos websites dos grupos hoteleiros/entidades de management permitem concluir que estes possuem algumas condições de acessibilidade, todavia estão longe do que é essencial para possuírem a total conformidade com as 
normas WCAG 2.0. Todos são inacessíveis, apresentam números elevados de "Erros" e "Avisos" nos pontos de verificação dos níveis de conformidade.

No que toca aos índices de acessibilidade, obtidos pela avaliação automática, verifica-se que o valor máximo corresponde ao Marriott Hotels \& Resorts, de 5.6 numa escala de 10 (Tabela 1). No entanto, através da avaliação manual e na análise a alguns itens, o número de erros aumentou significativamente em alguns dos casos e, nestas situações, o índice deveria ser inferior ao obtido automaticamente, podendo mesmo alterar a posição dos grupos hoteleiros/entidades de management. Tal verifica-se pela incapacidade da avaliação automática em analisar detalhadamente os itens do website e a presença de alguns atributos. Geralmente, se o website apresentar o atributo, mesmo que classificado incorretamente ou vazio, o item é validado e não é detetado como uma mais-valia em termos de acessibilidade. Assim, apenas na avaliação manual e mais cuidada torna-se possível detetar essas anomalias.

\section{Tabela 1: Índice de acessibilidade, por grupos hoteleiros/entidades de management, obtido através do AccessMonitor}

\begin{tabular}{|c|l|c|}
\hline $\begin{array}{c}\text { Posição do ranking } \\
\text { nacional (PRN) }\end{array}$ & \multicolumn{1}{|c|}{ Grupos hoteleiros/Entidades de management } & $\begin{array}{c}\text { Índice de } \\
\text { acessibilidade (IA) }\end{array}$ \\
\hline $\mathbf{5}^{\mathbf{0}}$ & Marriott Hotels \& Resorts & $\mathbf{5 . 6}$ \\
\hline $\mathbf{1}^{\mathbf{o}}$ & Pestana Hotels \& Resorts/Pousadas de Portugal & 4.9 \\
\hline $\mathbf{4}^{\mathbf{0}}$ & Tivoli Hotels \& Resorts & 4.8 \\
\hline $\mathbf{3}^{\mathbf{0}}$ & Accor Hotels & 4.3 \\
\hline $\mathbf{2}^{\mathbf{o}}$ & Vila Galé Hotéis & 3.3 \\
\hline
\end{tabular}

Fonte: Elaboração própria.

Aquando da análise dos itens (imagens, cabeçalhos, idioma e contraste de informação) apuraram-se diversas falhas na acessibilidade dos websites. Foram analisados, unicamente, os dados disponibilizados em cada item, não considerando informações e imagens cujos websites não os identificaram como tal, nos itens em análise. Numa fase inicial, foram verificadas as imagens detentoras de erros comprovados, como a inexistência de texto alternativo e a existência de texto sem qualquer significado. No item cabeçalho, o erro comum a todos os websites foi a falta de um cabeçalho principal, o primeiro na hierarquia de cabeçalhos, que facilita a navegação do utilizador. Em termos de idioma, na sua maioria, o mesmo é identificado, no entanto a não referência do idioma torna cada website inacessível a qualquer utilizador. Por fim, o item contraste de informação, que facilita a audição e a visualização de conteúdos aos utilizadores, não foi conseguida por nenhum website, sendo que apenas um apresentou informação sobre este item.

De acordo com os dados obtidos nas duas avaliações, e considerando a disponibilização de informação um fator determinante no processo de decisão de compra para o consumidor turístico, foi, ainda, analisada nos websites a existência de informação relevante para potenciais clientes portadores de necessidades especiais. No website do Pestana Hotels \& Resorts a informação sobre a acessibilidade física e a navegação do website não são de simples perceção, obrigando a uma pesquisa exaustiva até que o utilizador encontre a informação sobre as suas necessidades e preferências. A informação sobre a acessibilidade é generalizada e encontra-se na política de "Perguntas Frequentes". Relativamente aos serviços disponibilizados por cada hotel, a informação sobre a acessibilidade é observada de forma individual. O website do Vila Galé Hotéis apenas menciona informação sobre a acessibilidade para pessoas com mobilidade reduzida e, a mesma, é individualizada por cada hotel. O Accor Hotels apresenta no website de cada hotel um separador denominado "Serviços no hotel e nas proximidades", mencionando a existência de quartos acessíveis, de serviço de babysitter sob pedido e dos idiomas falados no hotel. Já o Tivoli Hotels \& Resorts nada apresenta no seu website e em cada hotel, de forma individualizada, qualquer informação sobre a acessibilidade para os seus potenciais clientes. Por fim, a informação sobre acessibilidade é disponibilizada de forma individual, por cada website do Marriott Hotels \& Resorts, no entanto, na "Visão geral" são retratadas as comodidades principais com detalhe sobre a "Acessibilidade". Ainda, relativa à mobilidade reduzida, os seus websites possuem imensos detalhes para o usufruto nas infraestruturas do hotel e dá a possibilidade de obtenção de mais informações, através de um contacto estabelecido com o gerente do hotel. 
Com isto, pode-se concluir que a disponibilização da acessibilidade nos websites não é um aspeto primordial para estes grupos hoteleiros/entidades de management. No estudo de Williams, Rattray e Grimes (2007), menciona-se que a falta de acessibilidade pode estar relacionada com a pouca sensibilidade dos webdesigners para tornarem as suas páginas acessíveis, além de equacionarem ver prejudicada a qualidade do trabalho e/ou aumentados os custos inerentes à criação e publicação do website. Pode, igualmente, atribuir-se ao desconhecimento dos benefícios competitivos, legais e tecnológicos que poderiam usufruir, ao satisfazerem as necessidades das pessoas com necessidades especiais.

\subsection{Recomendações}

As empresas devem estar conscientes do tipo de limitações ou incapacidades que mais dificultam o acesso à web, ou seja, se for motora ou dos membros superiores poderá estar relacionado com a utilização do rato ou do teclado, se for visual poderá apresentar dificuldade ou impossibilidade na leitura e se for auditiva poderá relacionar-se com a dificuldade ou impossibilidade na audição de vídeos ou de sons. No entanto, a resolução das barreiras de acessibilidade e usabilidade para uns utilizadores não devem criar problemas para os restantes. É nesta perspetiva que se apresentam algumas recomendações, consideradas essenciais, para garantir que os websites e os seus conteúdos beneficiem todos os cidadãos, especificamente as pessoas com necessidades especiais. No caso de se tratar da criação de um website, de raiz, as organizações devem cumprir o nível AAA dos critérios de acessibilidade das WCAG 2.0., conforme as recomendações W3C (2008). Para as organizações que já possuem um website, estas devem procurar saber qual o grau de acessibilidade de cada uma das páginas, podendo recorrer ao AccessMonitor, e devem melhorar a sua acessibilidade. A estrutura do website, os formulários e os conteúdos são algumas das principais áreas de atuação para o cumprimento das diretrizes de acessibilidade WCAG e recomenda-se alguns aspetos, nomeadamente uma fácil navegação através do teclado, do rato ou de outro produto técnico; uma apresentação lógica, clara e de linguagem simples de conteúdos; a informação em multiformatos, como por exemplo imagens descritas e vídeos legendados (Turismo de Portugal, 2017). Ainda, as diretrizes de acessibilidade WCAG (W3C, 2008) consideram que o fornecimento de indicações de navegação para localizar conteúdos, a criação de textos alternativos para conteúdo não textual e a facilitação de audição e visualização através do destaque dos elementos principais são fundamentais para a construção de websites acessíveis a todos os utilizadores. Tendo em conta os itens analisados neste estudo e as falhas detetadas, recomenda-se:

a) Imagem - as imagens devem ter um complemento alternativo textual colocado no atributo "alt", permitindo que a informação seja apresentada de várias formas a diversos utilizadores. Se o atributo "alt" não existir, as tecnologias assistidas serão incapazes de identificar a imagem e de transpor o seu significado ao utilizador.

b) Cabeçalhos - os cabeçalhos devem facilitar o processo de navegação das páginas e as empresas devem organizá-los de acordo com os níveis hierárquicos a que estão sujeitos. Em todas as páginas deve identificar-se um cabeçalho h1 (título principal da página) e os restantes servem para estruturar o site como se tratasse de um índice, com secções e subsecções.

c) Idioma - o código do idioma posiciona-se no elemento "html" com o atributo "lang" e/ou "xml:lang" e deve seguir o padrão de idiomas estabelecido, podendo ser composto por um código primário e por um subcódigo, separados por um hífen.

d) Contraste de informação - o contraste de informação é fundamental para garantir que o utilizador consiga aceder à página. Deve especificar-se as cores de primeiro plano e de fundo, combinando-as.

\section{Conclusões, limitações e investigação futura}

Sendo certo que o turismo está em franco desenvolvimento à escala mundial e que a evolução das TIC ocupam um papel diferenciador na indústria do turismo, a utilização de plataformas digitais, ajustadas a todo o tipo de consumidor, deve constituir uma prioridade para as empresas e instituições. O eTourism deve reger-se por princípios de igualdade e procurar eliminar as barreiras que colocam restrições às pessoas com necessidades especiais no acesso a todos os domínios da vida cultural, incluindo o turismo.

Tradicionalmente, a atividade turística envolvia uma complexa teia de relações, encadeadas e centradas na oferta turística. Atualmente, os operadores turísticos procuram atender às necessidades e preferências dos consumidores, sendo este o foco da sua atividade. Neste sentido, e com a massificação do uso das plataformas digitais, os consumidores tornaram-se mais informados, exigentes, atentos e críticos durante todo o processo de tomada de decisão. Por isso, o turismo teve de se ajustar e direcionar 
a sua estratégia de marketing para atender aos diferentes mercados, cada vez mais diferenciados e focalizados. Esta particularização da oferta turística dirige-se a segmentos de menor dimensão, os chamados nichos de mercado.

O turismo para portadores de necessidades especiais, considerado por muitos um nicho de mercado, apresenta hoje dados que merecem uma reflexão. Em Portugal 18\% da população apresenta dificuldades na execução de atividades básicas, na Europa o número atinge cerca de 17,6 \%, sendo que, à escala mundial os números não diferem muito. Se a estes números adicionarmos a população idosa, as crianças e os acompanhantes, os quais necessitam igualmente de contextos ajustados, este mercado poderá representar uma mancha muito significativa para o turismo. Assim, pode-se concluir que o turismo para portadores de necessidades especiais, em franco crescimento, representa um segmento de mercado com extremo potencial, no qual as empresas devem investir. Até porque, uma empresa que se preocupa em satisfazer este mercado, não só terá retorno a curto prazo como revela responsabilidade social e corporativa, contribuindo para a criação de um destino diferenciado, competitivo e sustentável.

Nesta perspetiva, entende-se que a criação de destinos turísticos acessíveis passa, igualmente, por um acesso à informação universal. Com as significativas alterações aos meios de comunicação e de informação, as empresas devem, ainda, ajustar-se na conceção das ferramentas digitais, de forma a conseguir incluir todas as pessoas no seu uso, eliminando as barreias existentes à comunicação e interação entre empresa e consumidor. Até porque, a divulgação online de um produto/serviço turístico, com a criação de uma página web, representa, muitas vezes, a porta de entrada a novos clientes. A má conceção dessas ferramentas digitais potencia a criação de novas barreiras e contribuem para a exclusão de pessoas no uso da web. Deste modo, se conclui que a acessibilidade na web poderá representar uma mais-valia para a sociedade em geral, pois potencia a inter-relação entre empresas e empresa-consumidor.

Com o estudo realizado, procurou-se perceber de que modo as organizações utilizam as ferramentas digitais para pessoas portadoras de necessidades especiais, tendo-se concluído que as organizações consideradas não cumprem os requisitos mínimos das diretrizes de acessibilidade WCAG, aspeto esse que aparentemente não constitui uma prioridade. As páginas web destas organizações expõem barreiras que impedem a inclusão digital. As empresas devem ter consciência do tipo de limitações ou incapacidades que mais dificultam o acesso à web, pois terão certamente perdas muito substanciais. $\mathrm{O}$ acesso universal, quer às páginas web quer às infraestruturas, potencia o aumento da procura, abrange todo o público-alvo da sociedade e reduz a sazonalidade. Posto isto, entende-se que deve haver uma maior consciencialização e esforço por parte das entidades reguladoras do setor do turismo, no sentido de estabelecer normas obrigatórias, de caráter universal e em consonância com os códigos internacionais, pois, só assim, se poderá construir uma sociedade moderna e verdadeiramente inclusiva.

No percurso efetuado foram surgindo algumas dificuldades e problemas que condicionaram a estruturação deste estudo, tendo sido necessário proceder ao reajustamento dos objetivos previamente delineados. Sentiu-se, também, lacunas no conhecimento das ferramentas automática e manual, no acesso à bibliografia em língua portuguesa e na escassez de tempo para aplicar uma entrevista, por forma a entender a predisposição das organizações para a utilização das plataformas digitais acessíveis e, também, para perceber a razão pela qual existe pouco investimento na disponibilização da informação física na web. Por isso, apesar da satisfação obtida pelo conhecimento adquirido, afirma-se aqui o carácter provisório das conclusões alcançadas, mesmo aquelas que aparentemente sugerem maior fiabilidade. Tais, representam o início de um longo percurso em torno da acessibilidade no eTourism, o qual se espera rigoroso e profícuo.

\section{Bibliografia}

Alexandre, D.

2012. O Turismo para Todos na oferta hoteleira de Lisboa: um custo ou um investimento? Dissertação de mestrado. Estoril: Escola Superior de Hotelaria e Turismo do Estoril.

Alles, M.

2009. Turismo accesible: importancia de la accesibilidad para el sector turístico. Entelequia: revista interdisciplinar, 9: 211-224. 
Altinay, Z., Saner, T., Bahçelerli, N., \& Altinay, F.

2016. The role of social media tools: Accessible tourism for disabled citizens. Journal of Educational

Technology \& Society, 19 (1): 89-99.

Assembleia da República

2009. Convenção sobre os Direitos das Pessoas com Deficiência. Resolução da Assembleia da República $n .^{\circ} 56 / 2009$. Diário da República n. ${ }^{\circ} 146 / 2009$, Série I de 30 de julho. Consultado a 14 de outubro de $2017 \mathrm{de} \mathrm{https://dre.pt/web/guest/pesquisa/-/search/493187/details/maximized.}$

Beck, U.

1991. Risk Society: Towards a New Modernity. London: Sage Publications.

Buhalis, D. (2000). Marketing the competitive destination of the future. Tourism Management, 21 (1): 97-116.

Buhalis, D., \& Law, R.

2008. Progress in information technology and tourism management: 20 years on and 10 years after the Internet - The state of eTourism research. Tourism Management, 29: 609-623.

Buhalis, D., \& Michopoulou, E.

2011. Information-enabled tourism destination marketing: addressing the accessibility market. Current Issues in Tourism, 14 (2): 145-168.

Buhalis, D., Darcy, S., \& Ambrose, I.

2012. Best practice in accessible tourism: Inclusion, disability, ageing population and tourism, 53. Channel View Publications.

Buhalis, D., Eichhorn, V., Michopoulou, E., \& Miller, G.

2005. Accessibility market and stakeholder analysis. University of Surrey y One Stop Shop for Accessible Tourism in Europe (OSSATE).

Buhalis, D., Leung, D., \& Law, R.

2011. eTourism: Critical information and communication technologies for tourism destinations. Destination marketing and management: Theories and applications, p. 205-224.

Castro, C.

2017. A Comunicação do Turismo Acessível: Um Estudo sobre as Atrações Turísticas da Cidade do Porto. Dissertação de mestrado. Porto: Faculdade de Economia da Universidade do Porto.

Chaffey, D., Ellis-Chadwick, F., Johnston, K. \& Mayer, R.

2006. Internet Marketing: Strategy, Implementation and Practice ( $3^{\text {rd }}$ Ed.). London: Pearson Education Limited.

comAcesso

2017. Ferramentas de validação automática. comAcesso. Site oficial, consultado a 26 de dezembro de 2017 de http://www.comacesso.pt/biblioteca/ferramentas-validacao-automatica.

Comissão Europeia

2010. Estratégia Europeia para a Deficiência 2010-2020: Compromisso renovado a favor de uma Europa sem barreiras. Bruxelas. Comissão Europeia.

Conforto, D., \& Santarosa, L.

2002. Acessibilidade à Web: Internet para todos. Informática na educação: teoria \& prática. Porto Alegre, 5 (2), p. 87-102.

Dalgic, T., \& Leeuw, M.

1994. Niche Marketing Revisited: Concept, Applications and Some European Cases. European Journal of Marketing, 28 (4), p. 39-55.

Darcy, S.

2001. People with physical disabilities and leisure. Celebrating inclusion and diversity in leisure, p. 59-80. Darcy, S., \& Dickson, T.

2009. A whole-of-life approach to tourism: The case for accessible tourism experiences. Journal of Hospitality and Tourism Management, 16 (1), p. 32-44.

Darcy, S., Ambrose, I., Schweinsberg, S., \& Buhalis, D.

2011. Conclusion: universal approaches to accessible tourism. Accessible tourism: Concepts and issues, p. 300-316. Bristol: Channel View Publications.

Darcy, S., Cameron, B., \& Pegg, S.

2011. Developing a business case for accessible tourism. Accessible tourism: Concepts and issues, p. 241-259. Bristol: Channel View Publications.

Deloite

2017. A arte do crescimento Atlas da Hotelaria 2017 - $12^{a}$ edição. Deloitte Consultores, S.A. 
$\mathrm{DN}$

2017. PE aprova 'lei da acessibilidade' para deficientes e aguarda aval dos Estados-membros. Diário de Notícias. Site oficial, consultado a 27 de dezembro de 2017 de https://www.dn.pt/lusa/interior/ pe-aprova-lei-da-acessibilidade-para-deficientes-e-aguarda-aval-dos-estados-membros-8770351.html.

Eichhorn, V., Miller, G., Michopoulou, E., \& Buhalis, D.

2008. Enabling access to tourism through information schemes? Annals of Tourism Research, 35 (1): 189-210.

El-Gohary, H.

2012. Factors affecting E-Marketing adoption and implementation in tourism firms: An empirical investigation of Egyptian small tourism organisations. Tourism management, 33 (5): 1256-1269.

ENAT

2017. European Network for Accessible Tourism. Site oficial, consultado a 28 de dezembro de 2017 de http://www.accessibletourism.org/.

Escobar-Rodríguez, T., \& Carvajal-Trujillo, E.

2013. An evaluation of Spanish hotel websites: Informational vs. relational strategies. International EU Journal of Hospitality Management, 33: 228-239.

2014. Web Accessibility. European Commission. Site oficial, consultado a 26 de dezembro de 2017 de $\mathrm{EU}$ http://ec.europa.eu/ipg/standards/accessibility/index_en.htm.

2017a. Press releases database. European Commission. Site oficial, consultado a 27 de dezembro de 2017 de http://europa.eu/rapid/press-release_IP-15-6147_pt.htm.

EU

2017b. Press releases database. European Commission. Site oficial, consultado a 27 de dezembro de $2017 \mathrm{de}$ http://europa.eu/rapid/press-release_IP-16-1654_pt.htm.

EUR-LEX Europe

2016. Diretiva (UE) 2016/2102 do Parlamento Europeu e do Conselho, de 26 de outubro de 2016, relativa à acessibilidade dos sítios web e das aplicações móveis de organismos do setor público. EUR-Lex Access to European Union law. Site oficial, consultado a 9 de dezembro de $2017 \mathrm{de} \mathrm{http://eur-lex.}$ europa.eu/legal-content/PT/TXT/?uri=CELEX\%3A32016L2102

Giddens, A.

1990. The Consequences of Modernity. Cambridge: Cambridge University Press.

Gonçalves, C.

2003. Enquadramento familiar das pessoas com deficiência: Uma análise exploratória dos resultados dos Censos 2001. Revista de Estudos Demográficos, 33, p. 69-94.

Gouveia, P., Mendes, D., \& Simões, J.

2010. Turismo Acessível em Portugal-lei, oportunidades económicas, informação. Instituto Nacional para a Reabilitação, I.P. Include, Coleção Informar, n. ${ }^{0} 7$.

Grupo Marktest

2017. 5,9 milhões de utilizadores de Internet em Portugal. Consultado a 29 de janeiro de 2018 de www. marktest.com/wap/a/n/id 22ba.aspx.

Grupo Marktest

2018. 4,4 milhões em sites de eCommerce. Consultado a 30 de janeiro de 2018 de http://www.marktest. $\mathrm{com} / \mathrm{wap} / \mathrm{a} / \mathrm{n} / \mathrm{id} \sim 234 \mathrm{~d}$.aspx.

Hoyo, M., \& Valiente, G.

2010. Turismo accesible, turismo para todos: la situación en Cataluña y España. Cuadernos de Turismo, 25 , p. 25-44.

INE

2012. Censos 2011. Instituto Nacional da Estatística. Site oficial, consultado a 29 de outubro de 2017 de http://www.ine.pt/xportal/xmain?xpid=INE\&xpgid=ine_destaques\&DESTAQUESdest_boui=107 $624784 \& D E S T A Q U E S t e m a=55466 \& D E S T A Q U E S m o d o=2$.

INR

1996. Inquérito Nacional às Incapacidades, Deficiências e Desvantagens. Instituto Nacional para a Reabilitação, I.P. Site oficial, consultado a 29 de outubro de 2017 de http://www.inr.pt/content/1/117/ estudos-informacao-estatistica. 
IRN

2018. Programas e Projectos. Instituto Nacional par a Reabilitação, IP. Site oficial, consultado a 15 de janeiro de 2018 de http://www.inr.pt/category/1/2/programas-projectos.

Li, X., Wang, Y., \& Yu, Y.

2015. Present and future hotel website marketing activities: Change propensity analysis. International Journal of Hospitality Management, 47, p. 131-139.

Michopoulou, E., \& Buhalis, D.

2011. Technology Platforms and Challenges. Accessible tourism: Concepts and issues, p. 287-299. Bristol:

Channel View Publications.

Michopoulou, E., \& Buhalis, D.

2013. Information provision for challenging markets: The case of the accessibility requiring market in the context of tourism. Information \& Management, 50 (5), p. 229-239.

Michopoulou, E., \& Buhalis, D.

2014. Usability requirements for accessible tourism systems. e-Review of Tourism Research (e-RTR).

Michopoulou, E., Darcy, S., Ambrose, I., \& Buhalis, D.

2015. Accessible tourism futures: the world we dream to live in and the opportunities we hope to have. Journal of Tourism Futures, 1 (3), p. 179-188.

Mills, J. E., Han, J. H., \& Clay, J. M.

2008. Accessibility of hospitality and tourism websites: a challenge for visually impaired persons. Cornell Hospitality Quarterly, 49 (1), p. 28-41.

Minghetti, V., \& Buhalis, D.

2009. Digital Divide in Tourism. Journal of Travel Research, XX (X), p. 1-15.

Montes, R., \& Aragall, F.

2009. Turismo Acessível ou Turismo para Todos? Sustentabilidade de Negócio. Revista Turismo \& Desenvolvimento, 11, p. 141-145.

Phillips, J., \& Peterson, H.

2001. Segmentation and Differentiation of Agri-Food Niche Markets: Examples from the Literature, staff paper. East Lansing, MI: Michigan State University.

Puhretmair, F., \& Nussbaum, G.

2011. Web design, assistive technologies and accessible tourism. Accessible tourism: Concepts and issues, p. 274-286. Bristol: Channel View Publications.

Robinson, M., \& Novelli, M.

2005. Niche tourism: an introduction. Niche tourism: contemporary issues, trends and cases, p. 1-11

Schlüter, R., \& Var, T.

1988. Resident attitudes toward tourism in Argentina. Annals of Tourism Research, 15 (3): 442-445.

Shuai, J., \& Wu, W.

2011. Evaluating the influence of E-marketing on hotel performance by DEA and grey entropy. Expert systems with applications, 38 (7): 8763-8769.

Stephen, A.

2016. The role of digital and social media marketing in consumer behavior. Current Opinion in Psychology, 10: 17-21.

travelBI

2018. Multi-National Travel Trends 2017. travelBI by Turismo de Portugal. Consultado a 30 de janeiro de 2018 de http://travelbi.turismodeportugal.pt/pt-pt/Documents/An\%C3\%A1lises/Mercados/multi-national-travel-trends-2017.pdf.

Turismo de Portugal

2017. Como ser um hotel acessivel. Site oficial, consultado a 15 de janeiro de 2018 de http://www. turismodeportugal.pt/Portugu\%C3\%AAs/turismodeportugal/all-for-all/Pages/como-ser-um-hotel-acessivel.aspx.

Turismo de Portugal

2018. Turismo Acessivel. Site oficial, consultado a 15 de janeiro de 2018 de http://www.turismodeportugal. $\mathrm{pt} /$ Portugu\%C3\%AAs/AreasAtividade/desenvolvimentoeinovacao1/Pages/turismoacessivel.aspx.

Unidade ACESSO

2017. AccessMonitor: Nota técnica. Unidade ACESSO. Consultado a 28 de dezembro de 2017 de http:// www.acessibilidade.gov.pt/accessmonitor/nota_tecnica.html. 
Vila, T., Darcy, S., \& González, E.

2015. Competing for the disability tourism market-a comparative exploration of the factors of accessible tourism competitiveness in Spain and Australia. Tourism Management, 47: 261-272.

Vos, S., \& Michopoulou, E.

2006. Inventory of accessibility schemes and data sets within the EU-tourism sector. EWORX S.A.: Shop for Accessible Tourism in Europe (OSSATE).

W3C

2017. Accessibility. World Wide Web Consortium. Site oficial, consultado a 22 de dezembro de 2017 de https://www.w3.org/standards/webdesign/accessibility.

WHO

2011. World report on disability. Geneva: World Health Organization. Consultado a 30 de outubro de 2017 de http://www.who.int/disabilities/world_report/2011/en/.

WHO

2017. What is e-accessibility? World Health Organization. Site oficial, consultado a 30 de outubro de 2017 de http://www.who.int/features/qa/50/en/.

Williams, R., Rattray, R., \& Grimes, A.

2007. Online accessibility and information needs of disabled tourists: A three country hotel sector analysis. Journal of Electronic Commerce Research, 8 (2), p. 157.

Xiong, L., Cobanoglu, C., Cummings, P., \& DeMicco, F.

2009. Website accessibility of US based hospitality websites. Information and communication technologies in tourism 2009, p. 273-284.

Yuksel, A., Yuksel, F. \& Bilim, Y.

2010. Destination attachment: effects on customer satisfaction, cognitive, affective and conative loyalty. Tourism Management, 31, 274-284.

\section{Webgrafia}

http://accessibleportugal.com/

http://www.accessibleTourism.org/?i=enat.en.accessible-cities

http://www.europeforall.com/home.seam

http://www.marriott.pt/default.mi

http://www.portugalacessivel.pt/default/home/id/1

https://www.accorhotels.com/pt/portugal/index.shtml

https://www.minorhotels.com/pt/tivoli

https://www.pestana.com/pt

https://www.pousadas.pt/pt

https://www.vilagale.com

https://www.visitportugal.com/pt-pt

\section{Notas}

\footnotetext{
Site oficial http://www.portugalacessivel.pt/default/home/id/1. Acedido a 25 de janeiro de 2018.

Site oficial https://www.visitportugal.com/pt-pt. Acedido a 25 de janeiro de 2018.

Site oficial http://accessibleportugal.com/. Acedido a 25 de janeiro de 2018.

Site oficial http://www.accessibleTourism.org/?i=enat.en.accessible-cities. Acedido a 25 de janeiro de 2018.

Site oficial http://www.europeforall.com/home.seam. Acedido a 25 de janeiro de 2018.
} 